\title{
Engagement associatif et cancer. Résultats d'une enquête sociologique
}

\section{Pour citer cet article :}

Knobé S., «Engagement associatif et cancer. Résultats d'une enquête sociologique », Bulletin du cancer, Vol.96, n ${ }^{\circ}$, mai 2009, pp.511-517.

\begin{abstract}
Résumé
L'espace associatif sida a fait l'objet de nombreuses recherches sociologiques s'intéressant tantôt au positionnement des différentes associations les unes par rapport aux autres, tantôt aux stratégies des acteurs engagés. La lutte contre le cancer, qui prend pourtant naissance pendant l'entre-deux-guerres, n'a de loin pas donné lieu à de tels développements. Ainsi, bien que le constat de l'absence, en France, d'une mobilisation collective et militante des patients atteints de cancer ait déjà été établi, les formes que prend l'engagement associatif des patients atteints de cancer n'ont pas été questionnées. Comment les malades s'engagent-ils au sein de l'espace associatif cancer? Nous essayerons d'y répondre par l'analyse de deux dimensions distinctes de l'engagement: les modes d'organisation des associations ainsi que les modes d'entrée des patients dans l'espace associatif.
\end{abstract}

Mots clés : association ; cancer ; patient

\section{Summary}

The associative anti-AIDS movement was the subject of many sociological research interesting in the various positioning of associations the ones compared to the others or in the strategies from the involved actors. The struggle against cancer, which however occurs during the inter-war period, did not give place to such developments. Thus, although the report of the absence, in France, of a collective and militant mobilization of cancer patients was already established, the forms of the cancer patients involment were not questioned. How do the cancer patients to get involved within the associative anticancer movement? We will try to answer this question by the analysis of two distinct dimensions from the involment: the type of association's organization and the type of patients' commitment the in the associative anticancer movement.

Key words : association; cancer; patient 


\section{Introduction}

L'espace associatif sida a fait l'objet de nombreuses recherches sociologiques s'intéressant tantôt au positionnement des différentes associations les unes par rapport aux autres, tantôt aux stratégies des acteurs engagés. La lutte contre le cancer, qui prend pourtant naissance pendant l'entre-deux-guerres, n'a de loin pas donné lieu à de tels développements. Alors que le constat de l'absence en France, contrairement aux Etats-Unis [1, 2], d'une mobilisation collective et militante des patients atteints de cancer a déjà été établi $[3,4,5]$, les formes que prend l'engagement associatif des patients atteints de cancer n'ont pas été questionnées. Leur étude éveille l'intérêt dans la mesure où peu d'associations se définissent publiquement comme des associations de patients. Comment s'y conçoit leur engagement? Quelles activités ou rôles leur sont dévolus ? La question de l'engagement individuel a fait l'objet de travaux aux orientations variées insistant tantôt, dans la lignée du paradigme du choix rationnel, sur l'intentionnalité de l'acteur, tantôt sur l'importance des déterminants structurels en termes de classe sociale et de réseaux sans toujours parvenir à une modélisation convaincante de cet engagement [6]. Le parti pris de porter une égale attention aux processus et à la dialectique permanente entre histoire individuelle et contextes sociaux [7] nous parait essentiel pour ce type d'analyse. L'articulation entre la structure des engagements possibles et les propriétés sociales des acteurs engagés permet de saisir les conditions dans lesquelles s'actualise cet engagement, en lien avec les interactions relatives aux positions et dispositions des acteurs associatifs environnants et de l'individu lui-même. Comprendre comment les personnes étant ou ayant été atteintes de cancer s'engagent dans l'espace associatif cancer nécessite donc d'analyser deux dimensions distinctes : les modes d'organisation des associations, en tant qu'elles définissent l'espace dans lequel s'effectue l'engagement, ainsi que les modes d'entrée des individus dans cet espace. Pour une plus grande clarté de l'exposé, dans les développements qui suivent l'appellation générique « malade » est utilisée indifféremment et sans connotation péjorative aucune pour désigner les personnes étant ou ayant été atteintes de cancer, c'est-à-dire qu'elles soient encre en traitement ou considérées en rémission. 


\section{Matériels et méthodes}

Nous avons procédé au recueil d'entretiens semi-directifs avec différents acteurs de l'espace associatif cancer. Deux principes directeurs ont guidé notre choix des associations à enquêter [cf. tableau 1]. D'une part, nous avons choisi de retenir des associations qui historiquement ont été pionnières dans la formalisation d'une aide aux malades, qui se caractérisent par une ancienneté de plus de trente ans, une implantation géographique qui couvre une grande partie du territoire national et qui occupent une position dominante dans l'espace (d'un point de vue symbolique et financier). Ces associations sont au nombre de quatre : la Ligue nationale contre le cancer (LNCC), l'Union des associations françaises de laryngectomisés et mutilés de la voix (UAFLMV), Vivre comme avant (VCA) et la Fédération des stomisés de France (FSF). D'autre part, nous avons cherché à prendre en compte des associations plus jeunes et présentant des actions innovantes. Ainsi, trois autres associations ont été enquêtées : Europa Donna - Forum France (EDFF), Jeunes solidarité cancer (JSC) et Essentielles.

Au total, 47 entretiens ont été réalisés avec des bénévoles en 2006-2007 [cf. tableaux 2 et 3]. L'échantillon est à $80 \%$ féminin et présente une moyenne d'âge de 54 ans. Bien que l'objectif de l'étude soit d'appréhender plus spécifiquement l'engagement associatif des malades à travers une lecture de leurs parcours en ce domaine, la compréhension des modes d'organisation des associations enquêtées nécessite aussi la prise en compte des points de vue d'acteurs non-malades qui se trouvent au nombre de dix et occupent essentiellement des fonctions d'administrateur. 


\section{Résultats et discussion}

Modes d'organisation des associations: une définition associative de l'engagement des malades

Au sein des associations investies dans l'aide aux malades, que celles-ci se définissent ou non comme des associations de patients, l'engagement ${ }^{1}$ des malades peut être soumis à des critères de sélection plus ou moins formalisés qui conditionnent, à des degrés divers, la possibilité même de leur engagement ou les rôles susceptibles de leur être dévolus. Une condition que nombre d'associations posent comme préalable à un engagement des personnes atteintes de cancer est la distance d'avec la maladie mesurée à l'aune de la durée qui les sépare de la fin de leurs traitements. L'idée sous-jacente est d'évaluer le passage du statut de «malade » à celui « d'ancien malade ». Certaines associations comme VCA ont institué leur mode de recrutement des bénévoles eu égard à ce type de critères, exigeant que trois à cinq ans se soient écoulés entre la fin des traitements et l'engagement associatif. L'évaluation de cette distance ne s'opère toutefois pas par rapport au seul critère objectif de durée écoulée depuis la fin des traitements mais consiste également en une interprétation psychosociale du rapport à la maladie. La future bénévole, outre son statut d'ancienne malade, doit être en mesure d'adopter le comportement jugé adéquat par VCA pendant les visites : par exemple, «laisser ses propres soucis et états d'âme au vestiaire » ou « ne pas parler de soi, écouter, ne pas juger, ne pas interpréter, être disponible »².

L'activité de visite des associations d'entraide (telles que VCA, I'UAFLMV et la FSF) tend à justifier un recrutement plus sélectif des malades, dont l'aptitude au bénévolat et le rapport à la maladie ont été évalués positivement. Pourtant, d'autres associations dont les modes d'action mises en œuvre ne semblent pas a priori nécessiter une telle évaluation de l'aptitude du malade à s'engager, au regard en particulier de sa «distance » d'avec la maladie, basent leur recrutement sur des principes similaires (LNCC ou EDFF par exemple).

\footnotetext{
1 Une précision s'impose quant à ce que recouvre le terme d'engagement associatif. Une première distinction s'établit déjà entre bénévole et bénéficiaire. Ce sont les premiers sur lesquels portent notre travail même si les deux s'entremêlent parfois. Une deuxième distinction est également à prendre en compte entre participants et adhérents, notre analyse portant sur les premiers. Ainsi, il s'agit de comprendre l'engagement des « malades-bénévoles-participants ».

${ }^{2}$ Le livret de la bénévole, VCA.
} 
Sans que ce recrutement soit formalisé de manière aussi précise qu'à VCA par exemple, il procède d'une sélection des malades selon le caractère positif de leur rapport à la maladie. Ainsi, le recul d'avec la maladie est censé tempérer, d'une certaine manière, l'attitude «agressive » ou « revendicative » de certains patients. Des associations créées plus récemment, comme JSC et Essentielles, nées du succès de la mise en place d'un forum de discussion sur Internet dédié, pour JSC, aux jeunes adultes atteints de cancer et, pour Essentielles, aux femmes atteintes de cancer du sein, n'adoptent pas ce type de critère de sélection. Au contraire, il y a une volonté explicite de permettre l'engagement quel que soit le moment du parcours de maladie.

Elaborer de manière plus ou moins formelle, le recrutement des malades participe à la définition des conceptions développées par chaque association de l'engagement légitime des malades. L'objet de l'association, son histoire mais aussi l'image qu'elle cherche à véhiculer dans l'espace public influencent ses prises de position non seulement quant au possible engagement des malades mais aussi quant à la forme que doit prendre leur encadrement. Ainsi s'opère aussi une distinction, pour ne pas dire une division, entre malades et non-malades. Certaines associations mettent de la sorte les malades à distance en ne leur permettant pas d'accéder aux fonctions à responsabilité au sein du conseil d'administration et encore moins du bureau. La LNCC ou EDFF comptent par exemple seulement l'un ou l'autre membre malade dont bien sûr la distance d'avec la maladie est très grande (en termes de durée). C'est la figure du malade représenté qui prédomine alors. Le pouvoir décisionnel est concentré essentiellement par des membres du corps médical.

Dans les associations d'entraide par contre, les malades occupent également des fonctions dirigeantes. Ainsi, le conseil d'administration est exclusivement ou en grande partie constitué de malades. Le pouvoir décisionnaire concernant autant le fonctionnement que les principales orientations à donner à l'association est entre les mains des malades euxmêmes. Lorsque ces associations comptent des salariés (généralement pour des travaux de secrétariat), il s'agit souvent d'anciens patients ayant au préalable été bénévoles (et continuant par ailleurs à participer bénévolement aux actions de l'association). Les plus 
anciennes de ces associations (I'UAFLMV, VCA et la FSF) comptent, parmi les membres de leur conseil d'administration, essentiellement « d'anciens » malades en correspondance avec la sélectivité préconisée par ces associations. A JSC et à Essentielles par contre, certains membres du bureau, constitué quasi exclusivement de malades, présentent une trajectoire de la maladie caractérisée par une durée longue et pouvant être qualifiée de trajectoire descendante [8] avec récidive(s) voire généralisation de la maladie. Toutefois, JSC, dont les activités d'entraide se complètent d'activités militantes, compte au sein de son conseil d'administration des personnes non-malades, personnalités du monde médical ou associatif, bien que le bureau soit uniquement composé de malades.

La manière dont les associations se positionnent les unes par rapport aux autres et formalisent les modes de recrutement et de participation des malades en leur sein contribue à définir les formes que peut revêtir l'engagement associatif des malades. La seule condition d'un diagnostic de cancer ne suffit pas à autoriser l'engagement dans certaines associations où des conditions de recul temporel d'avec la maladie et de modération des revendications sont plus ou moins fortement exigées, contribuant à circonscrire pour les malades l'espace des engagements possibles. Qui sont par conséquent ces malades qui s'y engagent? L'analyse plus spécifique des modes d'entrée dans l'espace associatif s'avère de fait nécessaire.

Modes d'entrée dans l'espace associatif: une définition sociale de l'engagement des malades

Bien entendu, un des premiers éléments susceptibles d'orienter le choix d'une association repose sur le type même de pathologie cancéreuse dont la personne est atteinte. Ainsi, une personne atteinte d'un cancer du larynx et une personne atteinte d'un cancer du sein n'envisagent pas nécessairement leur engagement dans la même association, si ce n'est dans une association "généraliste » comme la LNCC. L'orientation spécifique sur un type de pathologie peut parfois influencer la décision finale de l'engagement. Mais dans l'espace associatif cancer, pour une même pathologie, existent parfois plusieurs associations. Le 
cancer du sein est sans doute celui pour lequel le nombre d'associations existantes est le plus important. Le choix s'oriente alors en fonction d'une part des activités proposées et d'autre part de la plus ou moins grande sélectivité à l'entrée. Ainsi, VCA, EDFF et Essentielles, ciblées toutes sur le cancer du sein, recrutent leurs bénévoles à différents temps du cancer. Le recul imposé par VCA empêche un certain nombre de femmes atteintes de s'y investir. Elles peuvent alors s'orienter vers EDFF ou Essentielles.

La plus grande sélectivité mise en place par les associations privilégiant l'engagement d'un «ancien » malade semble recouvrir des attentes différenciées exprimant globalement un distinguo entre aider les autres et s'aider soi-même (avec bien sûr divers degrés d'entremêlement possibles). L'éloignement temporel d'avec la maladie apparaît en accord avec les activités de soutien développées par l'UAFLMV, VCA et la FSF dont la philosophie valorise l'image de l'ex-malade qui s'en est sorti, image également privilégiée par la LNCC. La conception d'une entraide qui entremêle aider les autres et s'aider soi-même, que l'on retrouve exprimée dans les attentes des bénévoles d'EDFF, de JSC et d'Essentielles, satisfait aussi les malades engagés encore en traitement. Ces associations favorisent surtout l'accès à des informations médicales, scientifiques ou juridiques, le partage d'expériences personnelles et, pour EDFF et JSC, la formalisation de revendications politiques. Les deux premiers points s'avèrent davantage recherchés en début de trajectoire de la maladie ou en cas de récidive. Notre enquête étant ponctuelle, il ne nous est toutefois pas possible d'évaluer quel peut être le turn-over de ces bénévoles eu égard à l'avancée de leur trajectoire de la maladie. En effet, dans ces associations au recrutement moins sélectif, l'engagement a peut-être tendance à répondre aux premiers temps du cancer puis à s'étioler ou à s'arrêter surtout dans le cas d'une trajectoire de la maladie ascendante [8].

Le diagnostic de cancer et tout ce qui l'accompagne (incertitude du pronostic, traitements plus ou moins invalidants ou mutilants...) constituent, il est vrai, le point de départ de l'engagement des malades dans l'espace associatif cancer ${ }^{3}$. Mais l'on peut toutefois se

\footnotetext{
${ }^{3}$ II est à signaler par exemple que nous n'avons pas rencontré de personnes découvrant leur cancer alors qu'elles auraient déjà été engagées dans la lutte contre le cancer.
} 
demander si ces malades qui décident de s'engager possèdent déjà un passé associatif (hors cancer) ou s'il s'agit plutôt de primo-bénévoles. Inscrire son cancer dans une démarche associative consistera tantôt à réorienter, tantôt à démarrer une carrière associative. Ainsi, le cancer peut représenter un facteur déclenchant un premier engagement associatif. Pour ces primo-bénévoles (qui représentent un peu moins de la moitié des malades interviewés), l'engagement associatif suit généralement de près le diagnostic de cancer et s'effectue alors le plus souvent la même année ou l'année suivante. La rupture biographique [9] induite par le diagnostic de cancer s'exprime, entre autres, par le démarrage d'une carrière associative. Les quelques années de recul (trois à cinq ans) que demandent certaines associations à leurs bénévoles limitent sans doute l'espace des possibles et expliquent peut-être en partie que ces primo-bénévoles soient souvent à l'initiative de la création de nouvelles associations (JSC ou Essentielles par exemple).

Si le cancer paraît dans certains cas être à l'origine du démarrage d'une carrière associative dans le domaine de la lutte contre le cancer, un peu plus de la moitié des malades bénévoles que nous avons interviewés ont un passé associatif à leur actif avant même leur diagnostic de cancer. Ils semblent alors reconvertir [10] dans ce domaine un capital associatif voire militant [11] acquis dans d'autres secteurs associatifs. Certains de ces « anciens » bénévoles mettent ainsi au service de la lutte contre le cancer des compétences acquises et/ou développées lors de leurs précédents engagements. Des dispositions acquises au cours de la socialisation secondaire au sein d'autres associations et relatives à l'entraide et à la valorisation de l'aide aux autres sont reconverties dans la lutte contre le cancer du fait d'avoir été soi-même touché. Ces dispositions sont à même, pour certains bénévoles, d'expliquer le choix de s'engager dans telle ou telle association de l'espace associatif cancer proposant des activités similaires à celles précédemment soutenues dans des associations hors cancer. A cette homologie de choix semble également correspondre une homogénéité du recrutement social des associations.

Sans prétendre dégager ici le profil social type du malade bénévole, il est possible de mettre en exergue l'existence d'un recrutement social différencié entre associations. Certaines 
variations sont directement corrélées aux données épidémiologiques concernant le cancer et notamment l'existence d'inégalités sociales tant d'un point de vue de l'incidence que de la mortalité. En effet, la survie après cancer est en moyenne plus basse dans les catégories socioprofessionnelles plus défavorisées, et ce, quelle que soit la localisation des cancers. L'incidence quant à elle est plus élevée dans les catégories socioprofessionnelles basses pour les cancers du poumon, des voies aérodigestives supérieures, de l'œsophage et du col utérin, et dans les catégories socioprofessionnelles élevées pour le cancer du colon et le cancer du sein [12]. De plus, les hommes et les femmes ne sont pas touchés dans les mêmes proportions par certains types de cancer. Le cancer des voies aérodigestives supérieures par exemple touche essentiellement les hommes, ce qui explique la très faible part d'adhérentes à I'UAFLMV. Quoi qu'il en soit le bénévolat en matière de lutte contre le cancer est incontestablement une affaire de femmes. Si l'on excepte les associations centrées sur des cancers féminins, il s'avère que même les associations "généralistes 》 comptent majoritairement des femmes bénévoles (JSC par exemple compte environ $10 \%$ d'hommes parmi ses bénévoles) ce que corrobore une étude sociologique récente [13].

L'engagement dans les associations de lutte contre le cancer les plus anciennes enquêtées ici (la LNCC, I'UAFLMV, VCA et la FSF) est davantage le fait de personnes sans profession (LNCC et VCA) ou ayant mis fin à leur activité professionnelle (UAFLMV et FSF). Les maris des femmes bénévoles sans profession exercent généralement une profession de cadre. II s'agit d'une forme de bénévolat qui n'est pas sans rappeler celui des « dames de la Ligue » du début du $20^{\text {ème }}$ siècle. Le bénévolat donne de la sorte à ces femmes une occupation sociale légitime compensant leur inactivité professionnelle. Ces femmes expriment d'ailleurs, de manière prépondérante, des attentes «désintéressées " d'aide aux autres. Seule I'UAFLMV présente un recrutement plus populaire de ses bénévoles, souvent d'anciens employés (à la retraite). Les associations de création plus récente (EDFF, JSC et Essentielles) se distinguent quant à leur recrutement social dans la mesure où plus souvent il s'agit de bénévoles exerçant une activité professionnelle (même s’ils connaissent une période d'arrêt maladie longue durée, ils envisagent la reprise de leur activité). On constate 
alors, comme dans le monde associatif en général [14], une sur-représentation des catégories moyennes salariées, avec des bénévoles exerçant surtout des professions intermédiaires, quelques fois des professions d'employé et plus rarement des professions de cadre. II est vrai que les revendications concernant l'accès plus facile à l'information et l'idée de relation de partenariat avec les médecins développées par ces associations sont davantage portées, dans le milieu associatif, par les membres des classes moyennes diplômées [15]. Un constat identique a été établi outre-Atlantique, aux Etats-Unis, à propos des militantes de la lutte contre le cancer du sein [16].

\section{Conclusion}

En articulant les deux dimensions que sont les modes d'organisation des associations et les modes d'entrée des bénévoles, nous avons pu mettre en évidence les conditions associatives et sociales qui sous-tendent les formes que prend aujourd'hui l'engagement associatif des malades atteints de cancer. Les associations les plus anciennes de l'espace associatif cancer tendent à définir comme « apte à s'engager » un malade « éloigné » de la maladie. Bien entendu, l'activité principale de visites dans les hôpitaux, développée par les associations d'entraide comme l'UAFLMV, VCA et la FSF, renforce leurs prises de position quant à la nécessité d'une distance d'avec son propre parcours de maladie, qui semble toutefois moins justifiée pour des associations comme la LNCC ou EDFF ne procédant pas à ce type de visites. L'une et l'autre adoptent pourtant des points de vue similaires, privilégiant l'engagement d'un malade « éloigné » de la maladie et qui, même «éloigné », n'occupe que très rarement des fonctions dirigeantes. Des associations plus récentes (JSC et Essentielles) prennent clairement position contre une quelconque forme de sélectivité en encourageant et en permettant l'engagement quel que soit le moment ou la pente de la trajectoire de la maladie.

Les modes d'entrée dans l'espace associatif cancer s'appréhendent au regard de ces cadres qui délimitent l'espace des engagements possibles pour chaque malade mais ne suffisent pas à les comprendre. En effet, à une définition associative de l'engagement s'ajoute une 
définition sociale également différenciée selon les associations considérées. A nouveau, se laisse entrevoir une distinction entre les associations les plus anciennes et les plus récentes. Les bénévoles des associations les plus anciennes sont majoritairement des femmes inactives, qu'elles soient à la retraite ou sans profession, ayant souvent une ou plusieurs expériences associatives à leur actif avant de s'engager dans la lutte contre le cancer. Seule I'UAFLMV compte surtout des bénévoles masculins du fait des spécificités épidémiologiques des cancers des voies aérodigestives supérieures. Les associations plus récentes (EDFF, JSC et Essentielles) recrutent leurs bénévoles parmi les femmes actives exerçant majoritairement des professions intermédiaires. Si à EDFF, une grande part des bénévoles a également démarré sa carrière associative bien avant le diagnostic de cancer et ce, dans des associations à vocation culturelle ou caritative, à JSC et à Essentielles par contre, il s'agit plus souvent de primo-bénévoles. Une certaine affinité élective semble opérer, homogénéisant le recrutement social des bénévoles au sein de chaque association.

L'ensemble des éléments mis en évidence dans cet article montre qu'il y a des différenciations graduelles entre les associations par rapport à la question de l'engagement des malades. Celles-ci concernent le degré d'éloignement d'avec la maladie et le degré de participation possible des malades aux fonctions à responsabilité. A ce positionnement graduel des associations se superposent des différences en termes de propriétés sociales des malades engagés (configurations pathologiques, attentes et catégories socioprofessionnelles différenciées et acquisition ou renforcement de dispositions à l'engagement associatif). Le recours à une analyse longitudinale de l'engagement des malades, en suivant l'évolution de leur carrière associative, permettrait sans doute d'affiner la compréhension des logiques d'engagement et de mettre à jour les éléments susceptibles d'encourager un engagement durable ou au contraire une défection précoce. 


\section{Remerciements}

Ce travail de recherche a été financé par l'Institut national du cancer dans le cadre d'une bourse post-doctorale. II a été réalisé sous la direction de Patrice Pinell, directeur de recherche à l'INSERM et responsable de l'équipe Sociologie du champ médical au Centre de sociologie européenne à Paris.

\section{Références}

1. Myhre J. The breast cancer movement: seeing beyond consumer activism. JAMWA 1999; 1, 29-31.

2. Kolker E. Framing as a cultural resource in health social movements: funding activism and the breast cancer movement in the US 1990-1993. Sociology of health \& illness $2004 ; 6,820-844$.

3. Ménoret $M$. Innovation génétique et cancer du sein : l'organisation des rapports entre professionnels et usagers en France et aux Etats-Unis. Innovations et sociétés $2002 ; 2$, 37-54.

4. Ménoret M. Prévention du cancer du sein : cachez ce politique que je ne saurais voir. Nouvelles questions féministes 2006 ; 2, 32-47.

5. Gaudillière JP. Mettre les savoirs en débat ? Expertise biomédicale et mobilisations associatives aux Etats-Unis et en France. Politix 2002 ; 57, 103-122.

6. Fillieule O, Péchu C. Lutter ensemble. Paris : L'Harmattan, 1993.

7. Fillieule $O$. Propositions pour une analyse processuelle de l'engagement individuel. Revue française de science politique 2001, 1-2, 199-217.

8. Ménoret M. Les temps du cancer. Paris : CNRS Editions, 1999.

9. Bury M. Chronic illness as biographical disruption. Sociology of health \& illness 1982; 2, 168-182.

10. Tissot S, Gaubert C, Lechien MH (dir). Reconversions militantes. Limoges: Presses Universitaires de Limoges, 2005.

11. Matonti F, Poupeau F. Le capital militant. Essai de définition. Actes de la recherche en sciences sociales $2004 ; 155,5-11$.

12. Herbert C, Launoy G. Les cancers. In : Leclerc A. et al. Les inégalités sociales de santé. Paris : La Découverte/INSERM, 2000, 239-250.

13. Bataille P. Un cancer et la vie. Paris : Balland, 2003.

14. Barthélemy M. Les associations dans la société française : un état des lieux. Paris : Cevipof, 1994.

15. Pinell P. Mouvement associatif. In Lecourt D (dir). Dictionnaire de la pensée médicale. Paris: PUF, 2004, 762-765.

16. Boehmer U. The personal and the political. Women's activism in response to the breast cancer and AIDS epidemics. New York: State University of New York Press, 2000. 


\section{TABLEAU 1 : description des associations enquêtées}

\begin{tabular}{|c|c|c|}
\hline ASSOCIATION & $\begin{array}{l}\text { Activités des } \\
\text { bénévoles } \\
\text { interviewés }\end{array}$ & $\begin{array}{c}\text { Formation des } \\
\text { bénévoles }\end{array}$ \\
\hline $\begin{array}{l}\text { LNCC [1918] }]^{4} \text { - Ligue nationale contre le cancer } \\
\text { L'aide à la recherche, la prévention, l'information du public et l'aide aux malades (information, soutien moral et psychologique, aides financières, } \\
\text { réflexions des malades par rapport aux politiques publiques de lutte contre le cancer). }\end{array}$ & $\begin{array}{l}\text { Information du public, } \\
\text { écoute téléphonique, } \\
\text { droits des malades. }\end{array}$ & $\begin{array}{l}\text { OUI } \\
\text { (connaissance de } \\
\text { l'association, écoute- } \\
\text { accueil). }\end{array}$ \\
\hline $\begin{array}{l}\text { UAFLMV [1958] - Union des associations françaises de laryngectomisés et mutilés de la voix } \\
\text { Le soutien, l'entraide, l'incitation à l'apprentissage d'une voix de remplacement, I'aide morale à la réinsertion et à la réadaptation familiale, sociale et } \\
\text { professionnelle, la défense des intérêts et des droits des laryngectomisés et autres mutilés de la voix, la vente d'accessoires et de dispositifs } \\
\text { spécifiques. }\end{array}$ & $\begin{array}{l}\text { Information du public, } \\
\text { visites de patients } \\
\text { hospitalisés. }\end{array}$ & NON \\
\hline $\begin{array}{l}\text { VCA [1975] - Vivre comme avant } \\
\text { Répondre, par la réalisation de visites dans les hôpitaux, à l'attente des femmes opérées ou traitées pour un cancer du sein en leur proposant le } \\
\text { concours d'anciennes patientes qui ont subi et surmonté des affections analogues. }\end{array}$ & $\begin{array}{l}\text { Visites de patientes } \\
\text { hospitalisées. }\end{array}$ & $\begin{array}{c}\text { OUl } \\
\text { (visites à l'hôpital, } \\
\text { écoute) }\end{array}$ \\
\hline $\begin{array}{l}\text { FSF [1976] - Fédération des stomisés de France } \\
\text { Informer et aider à assumer les divers handicaps qu'entraînent la présence d'une stomie et les exigences de son appareillage, informer et assister } \\
\text { les stomisés et leurs familles pour leur apporter aide et réconfort, informer les instances administratives concernées, trop peu au fait des problèmes } \\
\text { particuliers des stomisés, assurer un réseau d'entraide ou de secours par le biais de permanences et de contacts, directs ou téléphoniques, } \\
\text { promouvoir activement la prise en charge d'infirmières spécialisées en stomathérapie, favoriser la réinsertion dans le monde actif, visiter les futurs ou } \\
\text { récents stomisés en pré ou post-opératoires, soutenir et encourager la prévention }\end{array}$ & $\begin{array}{l}\text { Visites de patients } \\
\text { hospitalisés. }\end{array}$ & $\begin{array}{l}\text { OUl } \\
\text { (visites à l'hôpital, } \\
\text { écoute) }\end{array}$ \\
\hline $\begin{array}{l}\text { EDFF [1998] - Europa Donna Forum France } \\
\text { Obtenir le soutien et accroître la solidarité des femmes à travers toute l'Europe face au cancer du sein par : une meilleure formation et information du } \\
\text { public et des professionnels de santé ; un dépistage ou diagnostic précoce ; une offre optimisée de soins médico-chirurgicaux et psychologiques ; } \\
\text { une sensibilisation du public concernant cette maladie ; une promotion de la recherche dans ce domaine particulier de la médecine. }\end{array}$ & $\begin{array}{l}\text { Information du public, } \\
\text { entraide mutuelle, } \\
\text { soutien moral et } \\
\text { psychologique. }\end{array}$ & NON \\
\hline $\begin{array}{l}\text { JSC [2002] - Jeunes solidarité cancer } \\
\text { Briser l'isolement des jeunes adultes malades, anciens malades et de leurs proches par : le soutien par le forum de discussion sur Internet; } \\
\text { l'information par le site Internet, une série de guides et un carnet de bord ; la lutte contre la précarité socioprofessionnelle par un travail de fond en } \\
\text { collaboration avec de nombreuses associations. }\end{array}$ & $\begin{array}{l}\text { Information du public, } \\
\text { entraide mutuelle, } \\
\text { soutien moral et } \\
\text { psychologique, droits } \\
\text { des malades. }\end{array}$ & NON \\
\hline $\begin{array}{l}\text { Essentielles [2005] } \\
\text { Venir en aide aux personnes touchées par le cancer du sein, faciliter la mise en relation de ces personnes, les informer sur tous les aspects de la } \\
\text { maladie, leur apporter un soutien psychologique au travers de personnes qui sont ou ont été touchées par la maladie par la mise à disposition d'un } \\
\text { forum de discussion sur Internet. }\end{array}$ & $\begin{array}{l}\text { Information du public, } \\
\text { entraide mutuelle. }\end{array}$ & NON \\
\hline
\end{tabular}

${ }^{4}$ Entre crochets est précisée l'année de création. Pour les fédérations, souvent constituées dans un deuxième temps, l'année de création correspond aux premières associations créées et appartenant à la fédération. 
TABLEAU 2 : description des bénévoles interviewés ( $N=47$ )

\begin{tabular}{|c|c|c|c|c|c|}
\cline { 2 - 6 } \multicolumn{1}{c|}{} & \multicolumn{4}{c|}{ AGE } & \multicolumn{1}{c|}{} \\
\hline SEXE & -45 ans & 45 à 55 ans & 56 à 60 ans & +60 ans & TOTAL \\
\hline Homme & 2 & & 3 & 4 & 9 \\
\hline Femme & 7 & 13 & 9 & 9 & 38 \\
\hline TOTAL & $\mathbf{9}$ & 13 & 12 & 13 & 47 \\
\hline
\end{tabular}

TABLEAU 3 : bénévoles étant ou ayant été atteints de cancer $\underline{(N=37)}$

\begin{tabular}{|c|c|c|c|}
\hline \multicolumn{4}{|c|}{ Durée écoulée depuis l'annonce du diagnostique de cancer } \\
\hline - 3 ans & [3 à 10 ans] & [11 à 15 ans] & $+\mathbf{1 5}$ ans \\
\hline 16 & 10 & 6 & 5 \\
\hline
\end{tabular}

